Intensivmedizin

Anaesthesist 2020 - 69:316-322

https://doi.org/10.1007/s00101-020-00781-y

Online publiziert: 24. April 2020

(c) Springer Medizin Verlag GmbH, ein Teil von Springer Nature 2020

\section{Einleitung}

Die aktuelle Coronavirus-Krankheit 2019 (COVID-19)-Pandemie ist eine dynamische Situation mit therapeutischen Herausforderungen und logistischen Unwägbarkeiten. Ein Mangel an medizinischer Schutzausrüstung und Intensivrespiratoren ist je nach Wirkung der „Flatten-the-curve“-Maßnahmen zu erwarten. Das Londoner „Imperial College COVID-19 Response Team“ schätzt selbst mit erweiterten Maßnahmen zur Verminderung des Sozialkontakts, dass in Europa 2,5-5/1000 Einwohner im Verlauf einen Beatmungsplatz benötigen [18]. Erfahrungen aus unseren europäischen Nachbarländern zeigen zudem verheerende Folgen für die Patientenversorgung, falls keine klar definierten Kapazitätsplanungen und Ausweichstrategien verfügbar sind. Das Register der Deutschen Gesellschaft für Intensivund Notfallmedizin (DIVI) listet aktuell die Verfügbarkeit einer Intensivtherapie in deutschen Kliniken (https://www. intensivregister.de/\#/intensivregister).

Wie viele Intensivrespiratoren und Anästhesiebeatmungsgeräte in Deutschland bei einem exponentiellen Anstieg der Fallzahlen einsatzfähig sind, ist jedoch nicht bekannt. Zudem besteht bei vielen Ärzten und Pflegekräften eine hohe Unsicherheit bezüglich der Möglichkeiten und Limitationen verschiedener Gerätetypen.

Ziel dieser Übersichtsarbeit ist es, dem Leser einen Überblick über die konzeptionellen und technischen Unterschiede

Q. Notz · J. Herrmann · J. Stumpner - B. Schmid · T. Schlesinger · M. Kredel · P. Kranke · P. Meybohm · C. Lotz

Klinik und Poliklinik für Anästhesiologie, Universitätsklinikum Würzburg, Würzburg, Deutschland

\title{
Anästhesie- und Intensivbeatmungsgeräte: Unterschiede und Nutzbarkeit bei COVID-19-Patienten
}

verschiedener Typen von Beatmungsgeräten zu geben. Zudem werden in den sozialen Medien zuletzt vielfach beachtete unkonventionelle Beatmungskonzepte kritisch diskutiert.

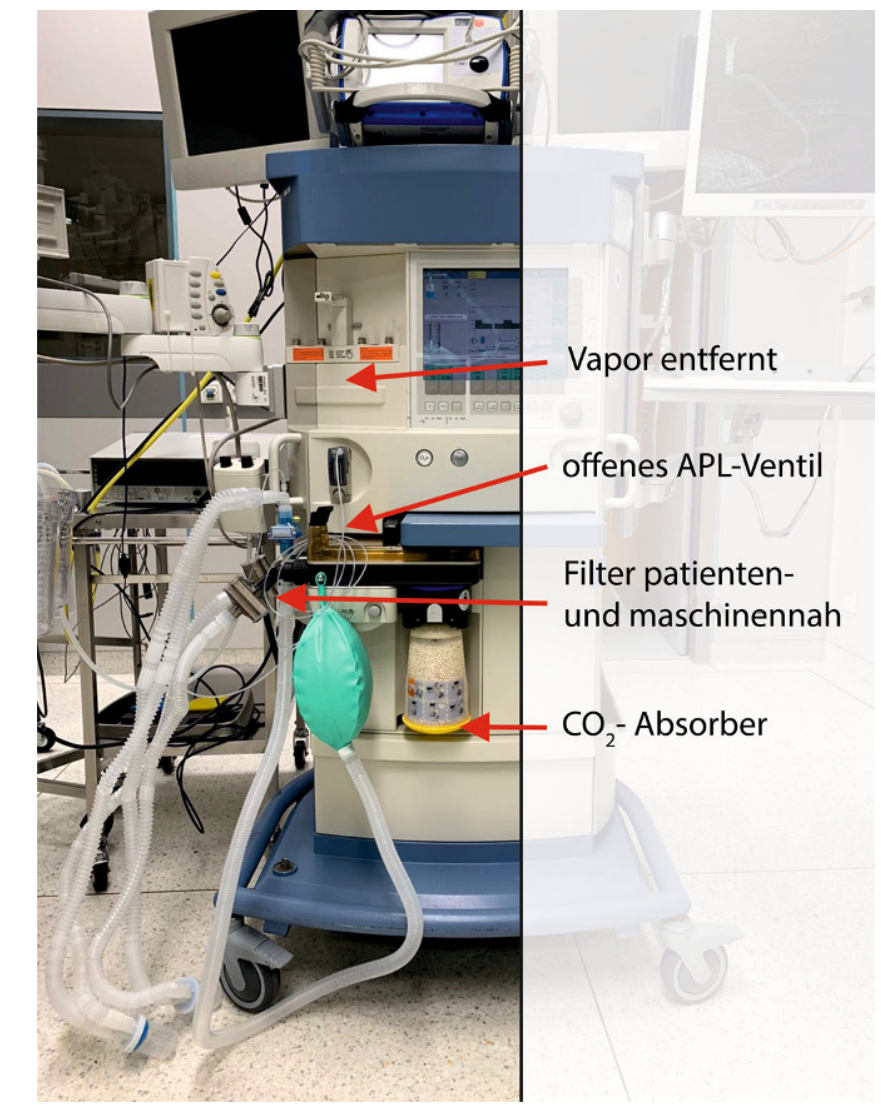

\section{Technische Voraussetzungen und Unterschiede}

\section{Intensivrespirator}

Intensivrespiratoren wurden speziell für die Behandlung von Patienten mit pathologischer Lungenmechanik entwickelt. Moderne Intensivrespiratoren sind mikroprozessorgesteuert und bieten eine
Abb. $1<$ Narkosegerät zur Langzeitbeatmung 
Tab. 1 Verwendung von Narkosegeräten zur Beatmung von intensivpflichtigen Patienten. (Modifiziert nach American Society of Anesthesiology [2]; Dräger persönliche Kommunikation)

\begin{tabular}{|c|c|c|c|c|c|}
\hline \multicolumn{6}{|c|}{ Verwendung von Narkosegeräten zur Beatmung von ARDS-Patienten } \\
\hline \multicolumn{6}{|l|}{ Geräteeinweisung, erfahrener Bediener } \\
\hline \multicolumn{6}{|c|}{ Notfall-Handbeatmungsbeutel vorhanden } \\
\hline \multicolumn{6}{|c|}{$\mathrm{CO}_{2}$-Absorber angebracht, keine $\mathrm{N}_{2} \mathrm{O}$-Versorgung angeschlossen } \\
\hline \multicolumn{6}{|c|}{ Vapore vom Anästhesiegerät entfernen, falls keine inhalative Sedierung erfolgen soll } \\
\hline \multicolumn{6}{|c|}{ Filter patienten- und maschinennah einbauen } \\
\hline \multicolumn{6}{|l|}{ Geräte-Kurz-Check vor Anwendung } \\
\hline \multicolumn{6}{|c|}{ Druckbegrenzungs(APL-)Ventil immer auf Position „offen“ stellen } \\
\hline \multicolumn{6}{|c|}{ Alarme auf voller Lautstärke, sinnvolle (enge) Alarmgrenzen (Cave: Leckagen, niedrige $\mathrm{F}_{1} \mathrm{O}_{2}$ ) } \\
\hline \multicolumn{6}{|c|}{ Enge Überwachung durch Anästhesisten, auf Frischgasdefizit achten } \\
\hline \multicolumn{6}{|c|}{ Bei hohen Flüssen > 5l/min Wärme- und Feuchtigkeitstauscherfilter (HME-Filter) verwenden } \\
\hline \multicolumn{6}{|c|}{ Bei Verwendung von Filtern kein Übertragungsrisiko für den nächsten Patienten. Sorgfältige Flächendesinfektion nach Beendigung der Beatmung } \\
\hline Vorgehen & Kontin. & Alle $1 \mathrm{~h}$ & Alle 4h & Alle $24 \mathrm{~h}$ & Alle $72 \mathrm{~h}$ \\
\hline Alarme & $\mathrm{x}$ & - & - & - & - \\
\hline $\begin{array}{l}\text { Kontrolle } \mathrm{CO}_{2} \text {-Absorber } \\
\text { Wechsel, wenn inspCO } \mathrm{CO}_{2}>3 \mathrm{~mm} \mathrm{Hg}\end{array}$ & - & $\mathrm{x}$ & - & - & - \\
\hline Kontrolle Kondensation, Wasserfalle & - & $x$ & - & - & - \\
\hline $\begin{array}{l}\text { Flusserhöhung, um System zu trocknen (v. a. } \\
\text { bei niedrigen Flüssen) }\end{array}$ & - & - & $x$ & - & - \\
\hline Filter wechseln (wenn durchnässt früher) & - & - & - & $\mathrm{x}$ & - \\
\hline $\begin{array}{l}\text { Selbsttest (ggf. überbrückend Transportre- } \\
\text { spirator) }\end{array}$ & - & - & - & $x$ & Ggf. $x$ \\
\hline
\end{tabular}

Vielzahl an Beatmungsmodi, inklusive diverser Spontanatmungsmöglichkeiten. Sie besitzen eine kompakte Bauweise und können so auch beim Transport der Patienten eingesetzt werden. Ebenso können eine assistierte nichtinvasive Beatmung (NIV) durchgeführt und Medikamente zur Atemtherapie verabreicht werden.

Die Geräte sind als offene Systeme ohne Rückführung der Ausatemluft konzipiert. Der Atemgasfluss und die eingestellte Sauerstoffkonzentration werden durch Ventile mittels eines „Flow-Zerhacker"-Prinzips gesteuert. Die schnell reagierenden Ventile zerlegen das Hochdruckgas in winzige Portionen und ermöglichen durch elektronische Steuerung die gewünschte Flussform und -stärke [15]. Weitere wichtige Merkmale sind in den meisten Modellen eine automatische Tubuskompensation für erleichterte Spontanatmung und eine automatische Leckagekompensation. Die Möglichkeit einer manuellen Beatmung mittels Atembeutel und Druckbegrenzungsventil existiert nicht.

\section{Anästhesiegeräte}

Narkosegeräte sind in der Regel halbgeschlossene Systeme und werden im Alltag mit niedrigen oder minimalen Frischgasflüssen betrieben. Die grundlegenden Charakteristika der Anästhesiegeräte betreffend Atemzugvolumen, Inspirationszeit, Beatmungsfrequenz, Inspirationsflow, Inspirationsdruck und positiv-endexspiratorischem Druck (PEEP) sind mit denen des Intensivrespirators vergleichbar. Auch die zur Verfügung stehenden volumen- und druckkontrollierten bzw. -regulierten Beatmungsmodi sind heutzutage ausreichend, um eine lungenprotektive Beatmung von ARDSPatienten sicherzustellen. In Bezug auf die Beatmungsmodi sind bei neueren Narkosegeräten (z.B. Perseus, Drägerwerk AG \& Co. KGaA, Lübeck) auch assistiert-kontrollierte und augmentiertspontanatmende Modi mit effektivem Triggering und Druckunterstützung problemlos möglich. Eine vergleichende Studie schlussfolgerte, dass aktuelle Narkosegeräte diesbezüglich ähnliche Leis- tungen wie Intensivbeatmungsgeräte liefern [10].

Trotz ausreichender technischer Voraussetzungen bestehen im Vergleich zu Intensivrespiratoren jedoch wesentliche konzeptionelle Unterschiede. Diese bedingen bei der Nutzung zur Langzeitbeatmung einen deutlich größeren Überwachungsaufwand mit der kontinuierlichen Anwesenheit eines Anästhesisten (- Abb. 1):

1. Im Unterschied zum Intensivrespirator findet eine Rückatmung des Ausatemgases statt. Das macht Narkosegeräte zum einen sparsamer als Intensivbeatmungsgeräte. Zum anderen reduziert die Rückatmung Wärme- und Flüssigkeitsverluste des Patienten. Das $\mathrm{CO}_{2}$ aus der Ausatemluft muss über spezielle Absorber eliminiert werden. Dieser sollte aus Sicherheitsgründen auch bei hohen Frischgasflüssen nicht entfernt werden.

2. Im Gegensatz zum Intensivrespirator besteht keine Frischgaskompensation. Die eingestellte $\mathrm{O}_{2}-\mathrm{Zufuhr}$ und der tatsächliche $\mathrm{O}_{2}$-Verbrauch 
stimmen beim Narkosegerät in Abhängigkeit von den Frischgasflüssen nicht unmittelbar überein. Die inspiratorische $\mathrm{O}_{2}$-Konzentration $\left(\mathrm{F}_{\mathrm{I}} \mathrm{O}_{2}\right)$ des Patienten muss engmaschig überwacht werden und der Frischgasfluss so angepasst werden, dass die gewünschte $\mathrm{F}_{\mathrm{I}} \mathrm{O}_{2}$ tatsächlich erreicht wird. Ein Frischgasdefizit ist bei Leckage jederzeit möglich und bedingt eine unzureichende Ventilation des Patienten.

3. Anästhesiegeräte bieten die Möglichkeit einer manuellen Beatmung unter Nutzung eines Druckbegrenzungsventils. Dieses sollte in der Langzeitbeatmung immer offen zur Raumluft sein, da die Geräte bei Versagen der kontrollierten Beatmung auf einen manuellen Beatmungsmodus wechseln. Bei geschlossenem Ventil und unbeabsichtigt hohem PEEP droht ein Kreislauf- bzw. akutes Rechtsherzversagen.

4. Bei Langzeitbeatmung sammelt sich Flüssigkeit in den Beatmungsschläuchen, die nicht nur Messungen verfälscht, sondern auch durch Schwingungen einen Atemversuch des Patienten simulieren kann. Hier wird von den Herstellern die Einstellung eines Frischgasflusses oberhalb des Atemminutenvolumens empfohlen, welches jedoch mit einem entsprechend hohen Gasverbrauch erkauft wird. Da dies wenig praktikabel scheint, sind bei niedrigen Flüssen eine regelmäßige Kontrolle auf Kondensationen und enge Alarmgrenzen obligat.

5. Die Nutzung der Vaporen zur Verabreichung volatiler Anästhetika wird von der Fa. Dräger (Drägerwerk AG \& Co. KGaA, Lübeck) und der Amerikanischen Gesellschaft für Anästhesiologie (American Society of Anesthesiology, ASA) für Langzeitbeatmung nicht empfohlen. Diese sollten vom Gerät entfernt werden [2]. Hintergrund ist eine versehentliche Applikation der volatilen Anästhetika durch unzureichende Überwachung oder unerfahrenes Personal. Dies sollte in Anwesenheit eines Anästhesisten jedoch kein Problem darstellen, und eine Nut-

Anaesthesist 2020 -69:316-322 https://doi.org/10.1007/s00101-020-00781-y

๑) Springer Medizin Verlag GmbH, ein Teil von Springer Nature 2020

Q. Notz · J. Herrmann · J. Stumpner · B. Schmid · T. Schlesinger · M. Kredel · P. Kranke · P. Meybohm · C. Lotz

\section{Anästhesie- und Intensivbeatmungsgeräte: Unterschiede und Nutzbarkeit bei COVID-19-Patienten}

\section{Zusammenfassung}

Die aktuelle Coronavirus-Krankheit 2019 (Covid-19)-Pandemie ist eine dynamische Situation mit therapeutischen Herausforderungen und logistischen Unwägbarkeiten. Ein Mangel an medizinischer Schutzausrüstung und Intensivrespiratoren ist je nach Wirkung der "Flatten-the-Curve" Maßnahmen zu erwarten. Gleichzeitig besteht bei vielen Ärzten und Pflegekräften eine hohe Unsicherheit bezüglich der Möglichkeiten und Limitationen verschiedener Gerätetypen. Intensivrespiratoren wurden speziell für die Behandlung von Patienten mit pathologischer Lungenmechanik entwickelt. Gleichwohl liefern aktuelle Narkosegeräte den Intensivbeatmungsgeräten ähnliche Leistungen und bieten meist viele Beatmungstypen inklusive unterstützender Spontanatmungsmodi. Trotz ausreichender technischer Voraussetzungen bestehen im Vergleich zu Intensivrespiratoren jedoch wesentliche konzeptionelle Unterschiede. Diese bedingen bei der Nutzung zur Langzeitbeatmung einen deutlich größeren Überwachungsaufwand. Moderne Transportbeatmungsgeräte eignen sich v.a. zur kurzfristen Überbrückung da bei Geräten mit Raumluftzufuhr immer eine Verwendung mit $100 \%$ Sauerstoff in kontaminierter Umgebung erfolgen sollte. In den sozialen Medien zuletzt vielfach präsentierte unkonventionelle Beatmungskonzepte zur Beatmung mehrerer Patienten mit einer Beatmungsmaschine, sog. "Ventilator-Splittung" ist nicht zu empfehlen. Ziel dieser Übersichtsarbeit ist es, dem Leser einen Überblick über die konzeptionellen und technischen Unterschiede verschiedener Typen von Beatmungsgeräten zu geben.

Schlüsselwörter

Covid-19 $\cdot$ Beatmung $\cdot$ Intensivmedizin

\section{Anesthesia and intensive care ventilators: differences and usability in COVID-19 patients}

\section{Abstract}

The current coronavirus disease 2019 (Covid19) pandemia is a highly dynamic situation characterized by therapeutic and logistic uncertainties. Depending on the effectiveness of social distancing, a shortage of intensive care respirators must be expected. Concomitantly, many physicians and nursing staff are unaware of the capabilities of alternative types of ventilators, hence being unsure if they can be used in intensive care patients. Intensive care respirators were specifically developed for the use in patients with pathological lung mechanics. Nevertheless, modern anesthesia machines offer similar technical capabilities including a number of different modes. However, conceptual differences must be accounted for, requiring close monitoring and the presence of trained personnel. Modern transport ventilators are mainly for bridging purposes as they can only be used with $100 \%$ oxygen in contaminated surroundings. Unconventional methods, such as "ventilator-splitting", which have recently received increasing attention on social media, cannot be recommended. This review intends to provide an overview of the conceptual and technical differences of different types of mechanical ventilators.

Keywords

Covid-19 · Mechanical ventilation · Intensive care zung nach Nutzen-Risiko-Abwägung erscheint somit im Einzelfall möglich. Die Lachgaszufuhr muss ebenfalls aus Sicherheitsgründen diskonnektiert werden.

6. Die Anästhesiegeräte verlangen alle 24 h einen Gerätesystemtest mit Diskonnexion vom Patienten und evtl. Neustart der Beatmungsmaschine.
Diese Zeit muss mit einem zweiten Beatmungsgerät überbrückt werden. Hier bietet sich beispielsweise der Einsatz eines Transportrespirators neuerer Generation an. Das Testintervall kann jedoch je nach Hersteller in der aktuellen Situation auf bis zu $72 \mathrm{~h}$ ausgedehnt werden. 


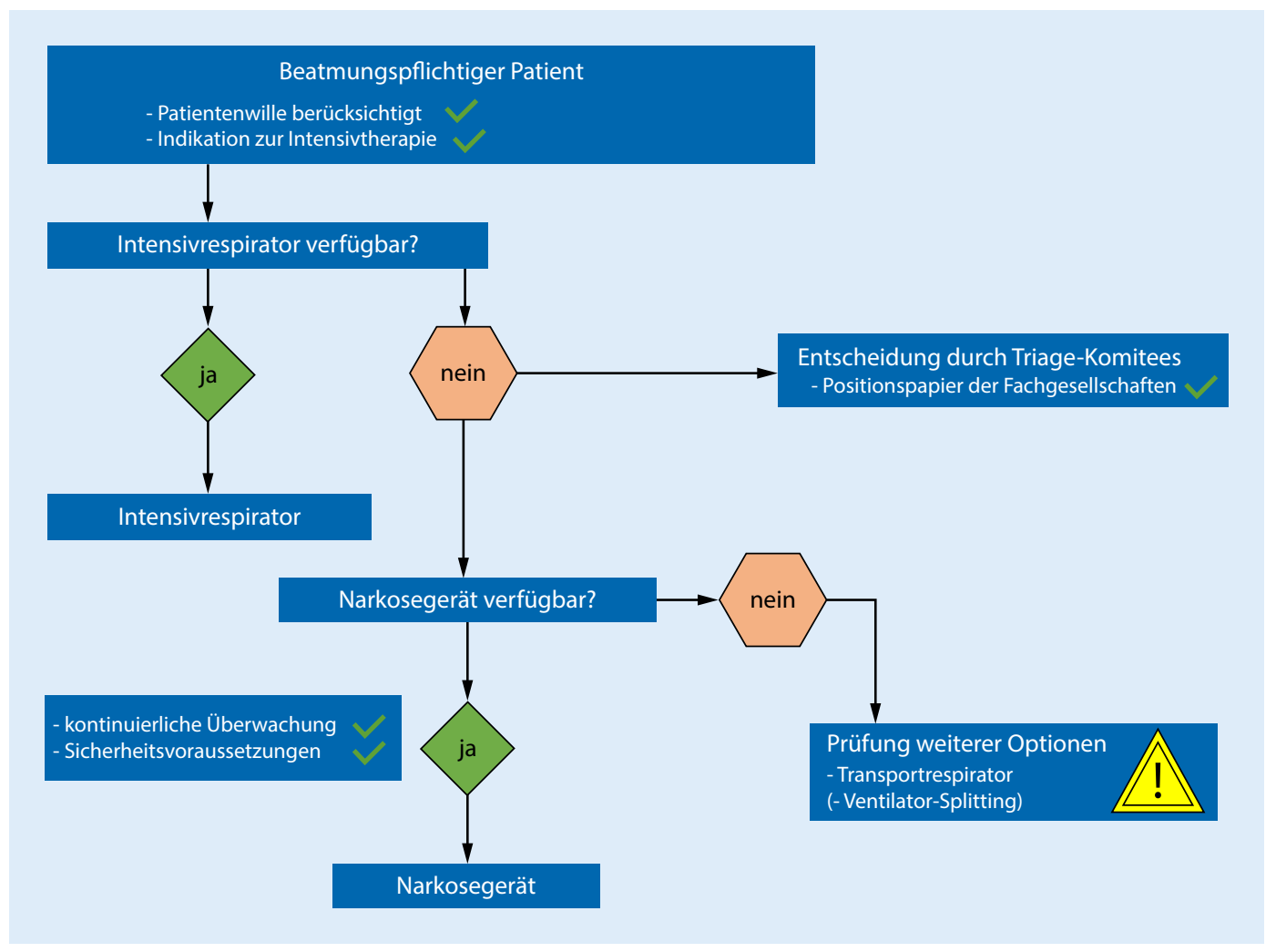

Abb. $2 \triangleleft$ Algorithmus zum differenzierten Einsatz von Beatmungsgeräten

Allgemein sind die veröffentlichten Empfehlungen der Hersteller zum Off-label use zu beachten. Aktuelle Informationen sind auf deren Homepages frei verfügbar und sollten vor dem Einsatz geprüft werden (• Tab. 1).

\section{Transportbeatmungsgeräte}

Beim Massenanfall von beatmungspflichtigen Patienten müsste auch auf Notfall- bzw. Transportrespiratoren zurückgegriffen werden. Diese unterscheiden sich sehr in Qualität und Ausstattung. Einfache pneumatisch betriebene Modelle bieten lediglich die Möglichkeit einer kontrollierten Beatmung mit $100 \%$ igem Sauerstoff, wohingegen neuere Generationen mit immer neueren Modi und ausgefeilterer Software ausgestattet sind. Turbinengetriebene Transportrespiratoren applizieren auch im ARDS-Setting (hohe Resistance bei niedriger Compliance) exakte Tidalvolumina. Es ist eine mit Intensivrespiratoren vergleichbare Leistung gegeben [4]. Die Systeme sind jedoch halboffen, d.h. bei längerer Beatmung ist/sind nicht nur der Frischgasverbrauch hoch, sondern auch
Flüssigkeits- und Wärmeverluste. Zudem ist bei Geräten mit Raumluftzufuhr, beispielsweise Oxylog-3000-Reihe (Fa. Drägerwerk AG \& Co. KGaA, Lübeck), in kontaminierter Umgebung immer $100 \%$ iger Sauerstoff zu verwenden. Ansonsten findet durch die Nutzung von Umgebungsluft eine Kontamination des Geräts statt [6]. Auch die Möglichkeiten des Monitorings der Beatmungsparameter sind im Vergleich zum Intensiv- oder zum Anästhesierespirator deutlich geringer. Somit sind diese Geräte kaum für die Langzeitbeatmung geeignet. Ein Einsatz zur Überbrückung etwaiger Gerätetests von Anästhesierespiratoren sollte jedoch ohne wesentliche Einschränkungen der Therapiequalität einhergehen.

\section{„Ventilator splitting“}

In den sozialen Medien ist in den letzten Wochen das Konzept des Ventilator splitting wiederentdeckt und viel diskutiert worden. In der Originalarbeit von 2006 zum Ventilator splitting von Neyman und Irvin wurden 4 identische und parallel geschaltete Kunstlungen zeitgleich von nur einem Intensivrespirator beatmet [12]. In volumen- und druckkontrollierten Modi konnten suffiziente Tidalvolumina erzielt werden. Dies wurde an Schafen über eine Dauer von $12 \mathrm{~h}$ experimentell bestätigt [13]. Zudem sind parallele Beatmungen an 2 gesunden Freiwilligen und an Neonaten in der Literatur beschrieben $[3,16]$. Insgesamt ist dieses Vorgehen jedoch kritisch zu sehen: Branson et al. zeichneten detaillierte Messreihen an Kunstlungen auf, die sich hinsichtlich Compliance und Resistance unterschieden. Die 4 Simulatoren mit je unterschiedlicher Compliance zwischen 50 und $70 \mathrm{ml} / \mathrm{cm} \mathrm{H}_{2} \mathrm{O}$ erhielten bei paralleler Ventilation stark abweichende Tidalvolumina zwischen 257 und $621 \mathrm{ml}$ [5]. Die Autoren schlussfolgerten, dass die parallele Ventilation mehrerer $\mathrm{Pa}$ tienten daher kein gangbares Konzept darstellt. Gerade im Zuge einer COVID19-Pneumonie dürften große Unterschiede hinsichtlich der Lungenmechanik, des individuellen $\mathrm{F}_{\mathrm{I}} \mathrm{O}_{2}$-Bedarfs und der PEEP-Werte zwischen den Patienten bestehen. Die Folge wären in Abhängigkeit von der individuellen Compliance hypo- oder hyperventilierte Patienten. Zusätzlich ist ein individuelles Moni- 
toring der Beatmungsparameter kaum möglich, sodass entsprechende Soll-IstAbweichungen sehr spät oder gar nicht erkannt würden. Trotzdem entwickeln Mediziner weltweit diesen Gedanken weiter. Nicht nur in der Gemeinschaft der Free Open Access Medical Education (FOAM) kursieren viele Beiträge $\mathrm{zu}$ diesem Thema $[8,14]$, die amerikanische Columbia University veröffentlichte bereits ein erstes Arbeitsprotokoll [9]. Gleichwohl sind solche Verfahren nicht zu empfehlen. Es besteht eine unzureichende Studienlage, und aufgrund der technischen Limitationen wären etwaige Strategien nur in einer verzweifelten Situation, nach Ausschöpfung aller regulären Beatmungsressourcen, zu rechtfertigen. Dies ist aktuell in Deutschland nicht zu erwarten.

\section{Automatisierung der Beutel- ventilation}

Ein weiterer Ansatz umfasst die Automatisierung der Beutelventilation durch einen Mikrocontroller/Mikroprozessor kontrollierten Elektromotor. Das Massachusetts Institute of Technology hat ein entsprechendes Gerät entwickelt und der FDA zur beschleunigten Zulassung vorgelegt. Tidalvolumen, Beatmungsdrücke, Atemfrequenz, das Inspirations:Exspirationsverhältnis und der PEEP sollen messbar und verstellbar sein. Als Notfallhilfe sind die Konstruktionsdaten für eine Zulassung durch die FDA als „open source“ frei verfügbar. Für Länder mit geringen finanziellen Ressourcen wäre dies eine kostengünstige Alternative zur manuellen Beatmung [1].

\section{Differenzierter Einsatz von Narkosegeräten zur Langzeitbeatmung}

Die Nutzung verschiedener (alternativer) Ressourcen ist automatisch auch mit der Frage einer Triage der Patienten vergesellschaftet. Die allgemein in der Medizinethik geltenden Handlungsgrundsätze „Nichtschadensprinzip“, „Gerechtigkeitsprinzip“ und "Fürsorgeprinzip“ gelten selbstverständlich auch für eine COVID-19-Pandemie. Eine Triage entlang dieser Grundsätze bedeutet für das behandelnde Klinikpersonal eine enorme ethisch-moralische Konfliktsituation und eine nicht $\mathrm{zu}$ unterschätzende psychische Belastung [17]. Hierbei ist zu beachten, dass ein „First-come-first-serve"-Ansatz nicht verfolgt werden sollte. In einer aktuellen Stellungnahme von Emanuel et al. wird empfohlen, die Triage von COVID-19-Patienten u.a. auf folgende übergeordnete Überlegungen zu stützen: Die Therapie sollte darauf ausgerichtet sein, möglichst viele Leben zu retten und Lebensjahre zu erhalten. Eine Gleichbehandlung unter Beachtung des instrumentellen Werts einer Person (z.B. medizinisches Personal) und Augenmerk auf die schlimmsten Fälle (z. B. junge Patienten, die viele Lebensjahre verlieren) ist durchzuführen. Ein "first come - first serve" würde diejenigen, die sich an die Vorschriften der Isolierung halten und erst später erkranken benachteiligen. Wichtig ist ebenso, nicht zwischen COVID-19- und Nicht-COVID19-Patienten zu unterscheiden. Knappe Ressourcen betreffen alle Patienten, unabhängig von deren Grunderkrankung [7]. Um die psychische Belastung der Triage auf mehrere Schultern zu verteilen, empfehlen Truog et al. die Implementierung von Triage-Komitees. Diese sollten aus erfahrenen Klinikern bestehen, die nicht in die Behandlung des Patienten involviert sind und so eine unabhängige Beurteilung und Abwägung treffen können. Wichtig ist, dass transparente Entscheidungen im Sinne einer expliziten Triage getroffen werden und eine implizite Triage vermieden wird. Hierzu gehört eine transparente und akkurate Kommunikation mit den Angehörigen [17]. Konkrete Handlungsempfehlungen für Deutschland wurden in einem gemeinsamen Positionspapier verschiedener Fachgesellschaften veröffentlicht. Besonderes Augenmerk wird dabei auf die patientenzentrierte Entscheidungsgrundlage gelegt, die den Patientenwillen und die Indikation zur Intensivtherapie umfasst. Es wird, soweit möglich, das grundsätzliche ethische Prinzip der "Autonomie“ berücksichtigt. Hierzu gehört insbesondere eine differenzierte Beurteilung des Patientenwillens und Gesundheitszustandes vor Aufnahme auf die Intensivstation.
Ein weiterer Aspekt ist eine regelmäßige Reevaluation des Krankheitsverlaufes [11].

\section{Fazit}

Die genaue Anzahl der in Deutschland zur Verfügung stehenden Beatmungsgeräte ist nicht bekannt. Gleichwohl bestehen im internationalen Vergleich hohe Kapazitäten. Hierbei kann nicht nur auf Intensivrespiratoren zurückgegriffen werden, sondern auch eine große Anzahl an Anästhesiegeräten genutzt werden. Diese sind eine wertvolle und unter Beachtung technisch-konzeptioneller Differenzen auch eine sichere Ressource zur Beatmung von ARDS-Patienten ( $\bullet$ Abb. 2). Auch moderne Transportbeatmungsgeräte bieten zur kurzfristigen Überbrückung eine sichere Beatmung von ARDS-Patienten. Dem Konzept der parallelen Ventilation ist hingegen nur mit großer Vorsicht zu begegnen. Es kann allenfalls nach Nutzen-RisikoAbwägung zum Einsatz kommen, falls die vorher genannten Ressourcen komplett ausgeschöpft sind.

\section{Korrespondenzadresse}

\section{PD Dr. C. Lotz}

Klinik und Poliklinik für Anästhesiologie, Universitätsklinikum Würzburg

Oberdürrbacher Str. 6, 97080 Würzburg, Deutschland

Lotz_C@ukw.de

\section{Einhaltung ethischer Richtlinien}

Interessenkonflikt. Q. Notz, J. Herrmann, J. Stumpner, B. Schmid, T. Schlesinger, M. Kredel, P. Kranke, P. Meybohm und C. Lotz geben an, dass kein Interessenkonflikt besteht.

Für diesen Beitrag wurden von den Autoren keine Studien an Menschen oder Tieren durchgeführt. Für die aufgeführten Studien gelten die jeweils dort angegebenen ethischen Richtlinien.

\section{Literatur}

1. https://e-vent.mit.edu/. Online abgerufen am 10. Apr. 2020

2. ASA (2020) Purposing anesthesia machines as ICU-ventilators. https://www.asahq.org/in-thespotlight/coronavirus-covid-19-information/ 
purposing-anesthesia-machines-for-ventilators. Zugegriffen: 31. März 2020

3. Bellini C, Risso FM, Serveli S et al (2013) Simultaneous transport of twin newborns. Air Med J32:334-337

4. BoussenS, GainnierM, MicheletP(2013)Evaluation of ventilators used during transport of critically ill patients: a bench study. Respir Care 58:1911-1922

5. Branson RD, Blakeman TC, Robinson BR et al (2012) Use of a single ventilator to support 4 patients: laboratory evaluation of a limited concept. respir care 57:399-403

6. Dräger (2020) COVID-19: Einsatz von Dräger Oxylog Notfall- und Transportbeatmungsgeräten zur Langzeitbeatmung. https://www.draeger.com/ Library/Content/COVID-19_Nutzung_Oxylog_ Langzeitbeatmung_Kundenschreiben.pdf.Zugegriffen: 31. März 2020

7. Emanuel EJ, Persad G, Upshur R et al (2020) Fair allocation of scarce medical resources in the time of Covid-19. NEngl J Med. https://doi.org/10.1056/ nejmsb2005114

8. Farkas J (2020) PulmCrit_-splitting ventilators to provide titrated support to a large group of patients. https://emcrit.org/pulmcrit/splitventilators/.Zugegriffen:31. März2020

9. Hospital CUCOPSNY-P (2020) Ventilator sharing protocol: dual-patient ventilation with a single mechanical ventilator for use during critical ventilator shortages. https://www.gnyha. org/wp-content/uploads/2020/03/VentilatorSharing-Protocol-Dual-Patient-Ventilation-witha-Single-Mechanical-Ventilator-for-Use-duringCritical-Ventilator-Shortages.pdf.Zugegriffen: 31 . März 2020

10. Jaber S, Tassaux D, Sebbane $M$ et al (2006) Performance characteristics of five new anesthesia ventilators and four intensive care ventilators im Druckure-support mode: a comparative bench study. Anesthesiology 105:944-952

11. Marckmann G, Neitzke G, Schildmann J et al (2020) Entscheidungen über die Zuteilung von Ressourcen in der Notfall und der Intensivmedizin im Kontext der COVID-19-Pandemie, S1-11

12. Neyman G, Irvin CB (2006) A single ventilator for multiple simulated patients to meet disaster surge. Acad Emerg Med 13:1246-1249

13. Paladino L, Silverberg $M$, Charchaflieh JG et al (2008) Increasing ventilator surge capacity in disasters: ventilation of four adult-human-sized sheep on a single ventilator with a modified circuit. Resuscitation 77:121-126

14. Pinson H (2020) A better way of connecting multiple patients to a single ventilator. https:// medium.com/@pinsonhannah/a-better-wayof-connecting-multiple-patients-to-a-singleventilator-fa9cf42679c6. Zugegriffen: 31. März 2020

15. Simon HB (2012) Anästhesiesysteme und Beatmungsgeräte. In: Rossaint R et al (Hrsg) Die Anästhesiologie

16. Smith R, Brown JM(2009) Simultaneous ventilation of two healthy subjects with a single ventilator. Resuscitation 80:1087

17. Truog RD, Mitchell C, Daley GQ (2020) The toughest triage - allocating ventilators in a pandemic. N Engl J Med. https://doi.org/10.1056/ nejmp2005689

18. Walker P, Whittaker C (2020) The global impact of COVID-19 and strategies for mitigation and suppression. https://www.imperial.ac.uk/ media/imperial-college/medicine/sph/ide/gidafellowships/Imperial-College-COVID19-GlobalImpact-26-03-2020v2.pdf. Zugegriffen: 31. März 2020

Koch, Thea, Heller, Axel R., Schewe, Jens-Christian (Hrsg.) Medizinische Einsatzteams

Prävention und optimierte Versorgung innerklinischer Notfälle, Scoringsysteme, Fallbeispiele

Berlin Heidelberg: Springer-Verlag 2019, 1. Auflage, 245 S., 55 Abb., (ISBN: 978-3662-58293-0), Softcover 39,99 EUR

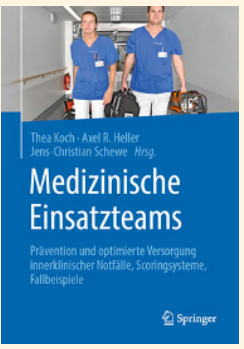

Die Letalität nach operativen Eingriffen ist trotz moderner chirurgischer Techniken und anästhesiologischer Verfahren höher als erwartet und beträgt nach jüngs-

ten Europäischen Daten 4\%. Daher ist es geboten, Konzepte zu entwickeln, um die perioperative Behandlungsqualität zu steigern. Ein Ansatz dabei ist die Einführung von Medizinischen Einsatzteams (MET), deren Aufgabe, im Gegensatz zu den klassischen Reanimationsteams, die Verhinderung von lebensbedrohlichen Ereignissen ist.

Bereits im Jahr 1997 wurden diese in den Niederlanden etabliert und es konnte eine signifikante Reduktion der Letalität, Aufnahmen auf Intensivstationen sowie Reanimationen nachgewiesen werden. Seither gibt es in zahlreichen Ländern Bestrebungen vergleichbare klinische Strukturen aufzubauen. Auch in Deutschland wurde eine Empfehlung zur Etablierung von MET zur Verbesserung der Behandlungsqualität von den anästhesiologischen und den chirurgischen Fachgesellschaften erarbeitet.

Die Herausgeber des vorliegenden Werkes legen nun das erste deutschsprachige Buch zu dieser Thematik vor, in dem in drei übergeordneten Sektionen alle wesentlichen Aspekte des Themenkomplexes in strukturierter und übersichtlicher Form dargestellt werden. Zunächst werden in anschaulicher Weise die Ziele und Möglichkeiten der MET präsentiert, im zweiten Kapitel die Fragen nach der Organisation und Schulung erörtert und Antworten gegeben, wie diese neue Konzeption umgesetzt werden kann. Letzteres ist insbesondere daher von großer Wichtigkeit, da sich in der Praxis häufig gezeigt hat, dass sinnvolle Projekte aufgrund einer mangelhaften Umsetzungsstrategie scheiterten. Im abschließenden Kapitel veranschaulichen Fallbeispiele aus unterschiedlichen Fachgebieten praxisnah, welchen Betrag MET zur optimierten Versorgung der Patienten leisten können.

Das Werk "Medizinische Einsatzteams" ist informationsreich und dabei exzellent geschrieben. Die Konzeption folgt einem interdisziplinären sowie auch interprofessionellen Ansatz und richtet sich damit gleichermaBen an Pflege- und Notfalldienstkräfte als auch Ärzt*innen unterschiedlicher Fachdisziplinen. Damit ist es den Herausgebern gelungen sowohl den umfangreichen Stoff einer breiten Leserschaft zugänglich zu machen als auch das Interesse zu wecken, diese innovative Struktur in die Klinik zu implementieren.

Zusammenfassend ist das Buch eine unverzichtbare Lektüre für alle diejenigen, die an der klinischen Patientenversorgung beteiligt sind.

F. Wappler (Köln) 
Hier steht eine Anzeige.

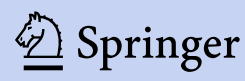

